\title{
Room Temperature based Fan Speed Control System using Pulse Width Modulation Technique
}

\author{
Vaibhav Bhatia \\ Department of Electrical and Electronics Engg., \\ Bhagwan Parshuram Institute of Technology, \\ New Delhi-110089, India
}

\author{
Gavish Bhatia \\ Department of Computer Science \& Engg., \\ Amity School of Engg. \& Technology, \\ Noida, India
}

\begin{abstract}
This paper presents the design and simulation of a novel fan speed control system based on room temperature using Pulse width Modulation Technique. The duty cycle is made to vary according to the room temperature and the fan speed is controlled accordingly. This paper elucidates how the autonomous speed control of fan is done based on data from the temperature sensor. The design proposed here is appropriate according to the modern lifestyle. The simulation of the system has been done on Proteus Professional Software v 8.0 and the various graphs showing relationship between temperature and different parameters have been plotted in MATLAB R2013a v8.1 to validate the accuracy of the system.
\end{abstract}

\section{General Terms}

Simulation, Temperature, Speed, Fan

\section{Keywords}

Microcontroller, Pulse Width Modulation (PWM), Temperature Sensor, Duty Cycle, Liquid Crystal Display (LCD)

\section{INTRODUCTION}

With the advancement in technology, intelligent systems are introduced every day. Everything is getting more sophisticated and intelligible [1-2].There is an increase in the demand of cutting edge technology and smart electronic systems. Microcontrollers play a very important role in the development of the smart systems as brain is given to the system. Microcontrollers have become the heart of the new technologies that are being introduced daily. A microcontroller is mainly a single chip microprocessor suited for control and automation of machines and processes. Today, microcontrollers are used in many disciplines of life for carrying out automated tasks in a more accurate manner [3-5]. Almost every modern day device including air conditioners, power tools, toys, office machines employ microcontrollers for their operation [6-8]. The block diagram of the microcontroller system is shown in Fig 1.

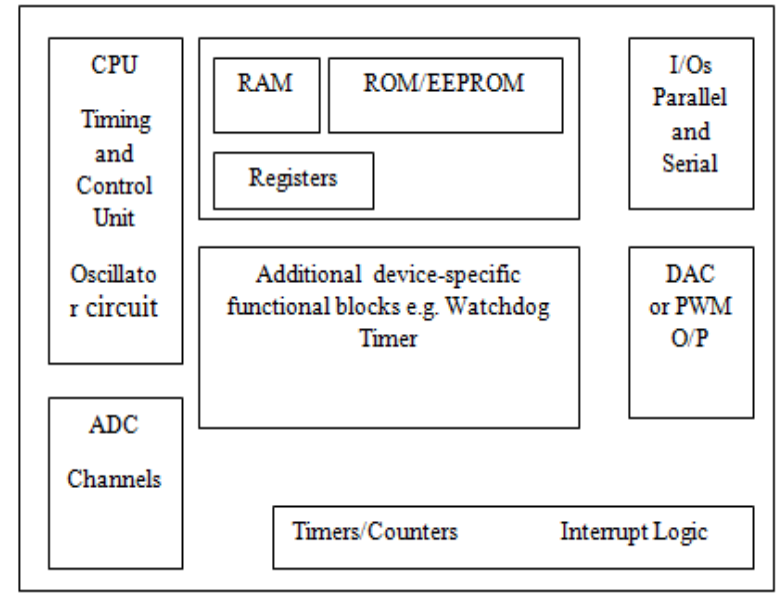

Fig.1. Microcontroller System

Microcontroller essentially consists of Central Processing Unit (CPU), timers and counters, interrupts, memory, input/output ports, analog to digital converters (ADC) on a single chip [9]. With this single chip integrated circuit design of the microcontroller the size of control board is reduced and power consumption is low [10]. This paper presents the design and simulation of the fan speed control system using PWM technique based on the room temperature. A temperature sensor has been used to measure the temperature of the room and the speed of the fan is varied according to the room temperature using PWM technique. The duty cycle is varied from 0 to 100 to control the fan speed depending upon the room temperature, which is displayed on Liquid Crystal Display.

\section{PULSE WIDTH MODULATION}

Pulse Width Modulation (PWM) is a technique in which the width of the periodic sequence pulses is varied in accordance with the baseband signal. PWM is also known as Pulse Duration Modulation. The leading edge of the pulse is held constant and the change in pulse width with signal is measured with respect to leading edge. In PWM, the pulse width is proportional to the amplitude of the signal. By varying the duty cycle of the pulse, the speed of the fan can be controlled. Duty cycle may be defined as the amount of time in a particular period during which the pulse is active or high. The speed is made slow, medium, fast, very fast and zero by having different duty cycles. Fig 2 shows the pulses with varying duty cycles: 


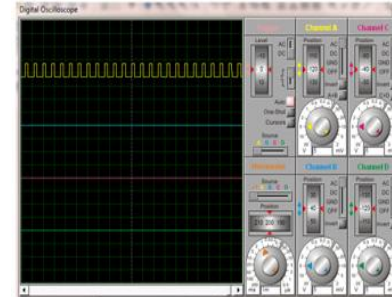

Duty Cycle-25\%

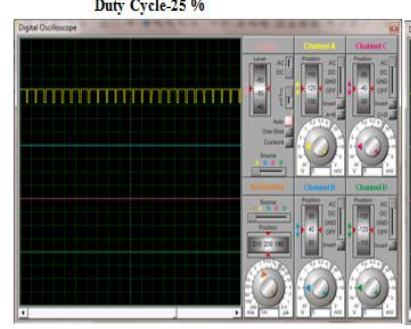

Duty Cỵcle-75\%

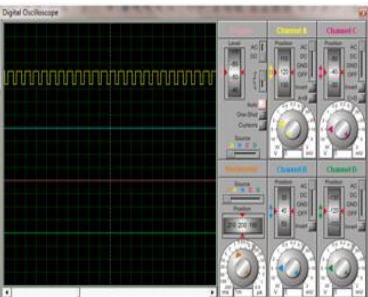

Duty Cycle-50 \%

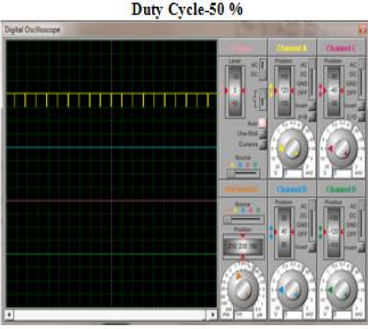

Duty Cycle-100\%
Fig.2. Different Duty Cycles

\section{BLOCK DIAGRAM}

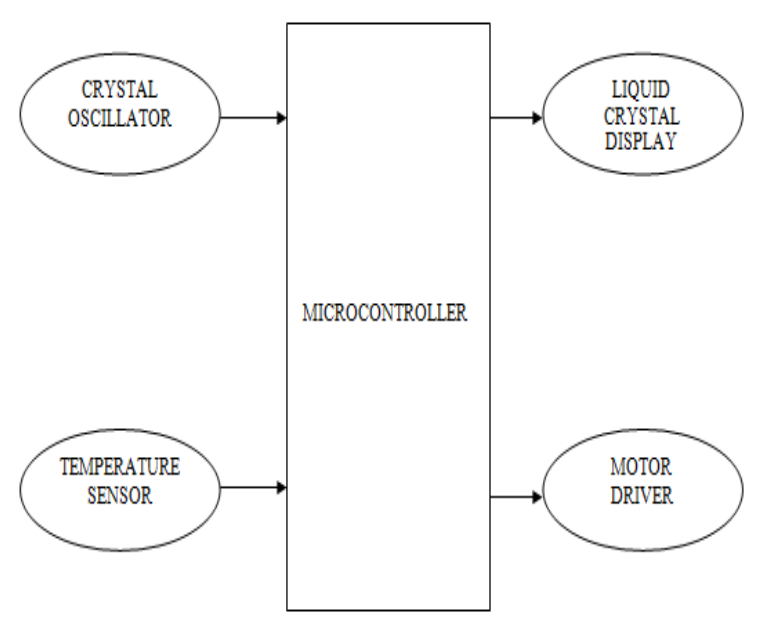

Fig.3. Block Diagram

The block diagram of the system has been shown in Fig 3 .It consists of:

1. Crystal Oscillator- A crystal oscillator is an oscillator circuit, which uses the mechanical resonance of a vibrating to create an electrical signal with a very precise frequency. The crystal oscillator is used in the system to provide clock signal to the microcontroller.
2. Temperature Sensor- The LM35 is a precision IC temperature sensor, whose output voltage is proportional to the temperature in Celsius. LM35 sensor is interfaced with the microcontroller to measure the room temperature.

3. Liquid Crystal Display- The LCD is a dot matrix liquid crystal display that displays alphanumeric characters and symbols. 16X2 LCD digital display has been used in the system to show the room temperature.

4. Motor Driver- Motor driver IC is used to control motors through a microcontroller. L293D IC is used as a motor driver IC. It provides different logic to control the direction of the motor. It is assembled in a 16 pin lead plastic package, which has 4 center pins connected together.

\section{POWER SUPPLY}

Five volts power supply w.r.t ground is needed for the operation of the microcontroller. Fig 4 shows the schematic of the power supply used by the microcontroller.

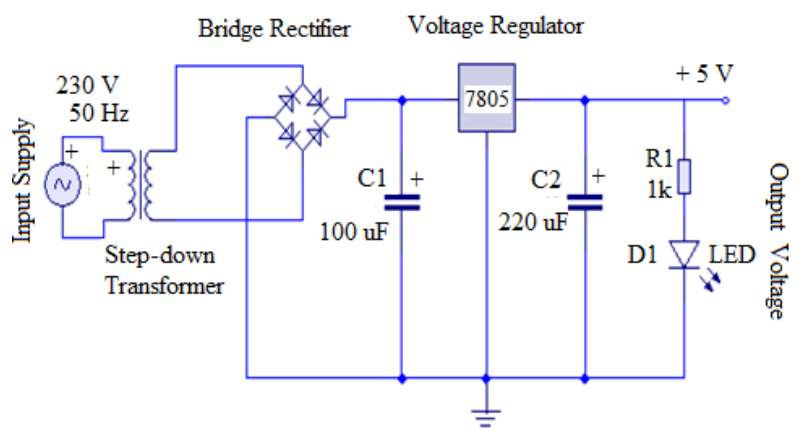

Fig.4. Power Supply Schematic

The power supply consists of a step down transformer which is used to convert $230 \mathrm{~V}, 50 \mathrm{~Hz}$ AC voltage to $12 \mathrm{~V} \mathrm{AC}, 50$ $\mathrm{Hz}$. This $12 \mathrm{~V}$ AC voltage is given to the bridge rectifier, which converts it into $12 \mathrm{~V} \mathrm{dc}$. A voltage regulator 7805 is used to convert the $12 \mathrm{~V}$ dc into $5 \mathrm{~V}$ dc which is needed by the microcontroller. Capacitors are used for smoothening the output voltage. This five volts dc thus produced is given to the microcontroller for its operation.

\section{CIRCUIT SIMUlation}

The simulation of the system has been done on Proteus Professional Software v8.0. PIC16F877A microcontroller based on Modified Harvard architecture is used in the system. Coding of the system has been done in Embedded $\mathrm{C}$ language. $16 \mathrm{X} 2 \mathrm{LCD}$ display has been used which is connected to PORT B of the microcontroller. The simulation of the circuit is shown in Fig 5: 


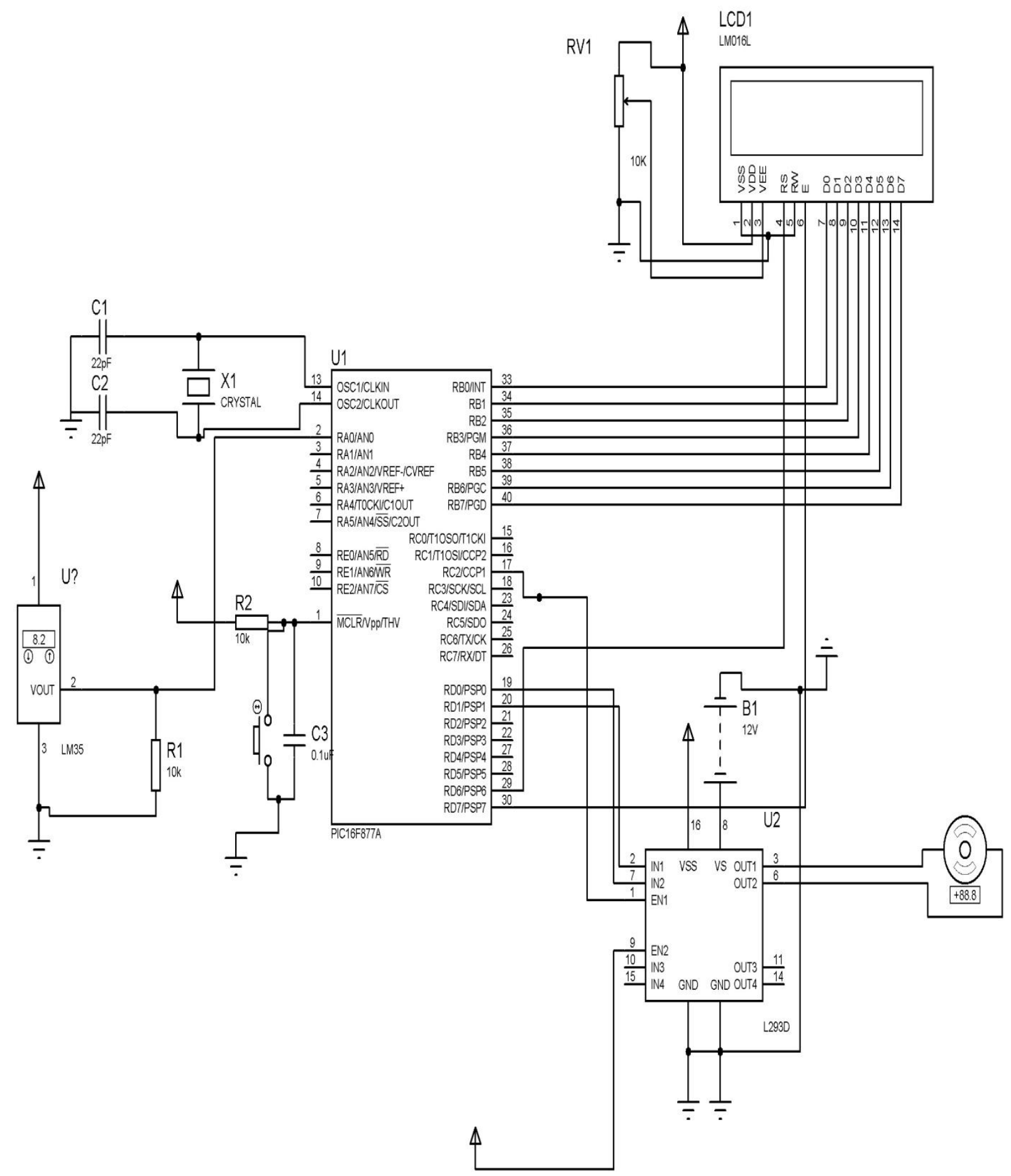

Fig.5. Circuit Simulation 
The temperature sensor senses the room temperature and it is displayed on the LCD. The speed of the fan is controlled by using PWM technique according to the room temperature. For processing analog signals, microcontroller has analog to digital converter which converts analog signals to digital ones. The temperature sensor LM 35 interfaced to the analog port acquires the room temperature and converts it into digital voltage signal. Fig 6 shows the relationship between digital voltage and the temperature.

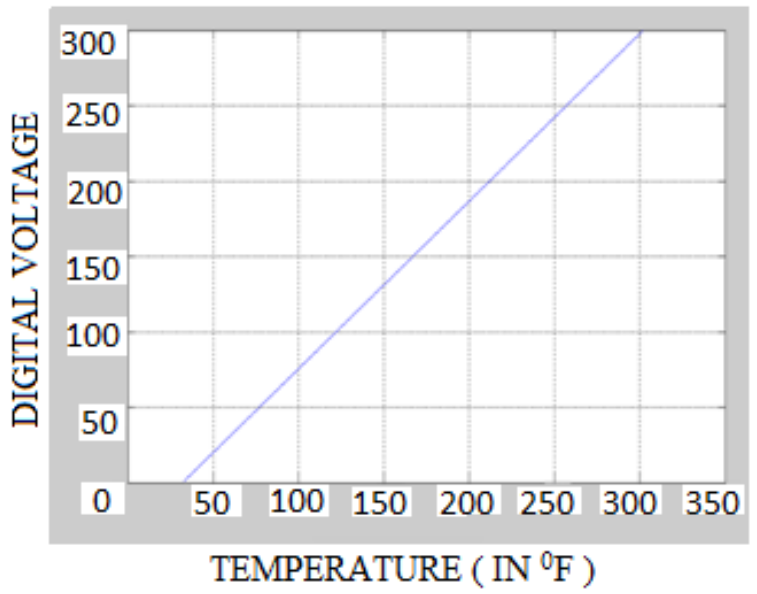

Fig.6. Digital Voltage vs. Temperature (in Fahrenheit)

The microcontroller used in this system has inbuilt PWM module which is used to control speed of the fan by varying the duty cycle. According to the readings from the temperature sensor duty cycle is varied automatically thus controlling fan speed. Table 1 shows the duty cycles varying with the temperature.

Table 1: Duty cycle and Temperature

\begin{tabular}{|c|c|c|c|}
\hline S.no & $\begin{array}{c}\text { Temperature } \\
\left(\operatorname{In}{ }^{\circ} \mathrm{C}\right)\end{array}$ & $\begin{array}{c}\text { Duty Cycle } \\
(\%)\end{array}$ & Speed \\
\hline 1. & Less than 0 & 0 & Zero \\
\hline 2. & 0 to 10 & 25 & Slow \\
\hline 3. & 10 to 20 & 50 & Medium \\
\hline 4. & 20 to 30 & 75 & Fast \\
\hline 5. & 30 to 40 & 100 & Very Fast \\
\hline 6. & Greater than 40 & 100 & Very Fast \\
\hline
\end{tabular}

The medium speed corresponds to 50 percent duty cycle and very fast corresponds to 100 percent duty cycle. The variation of the duty cycle with temperature (in Celsius) is shown in the Fig 7. The fan is in full swing when the duty cycle is made 100 percent.

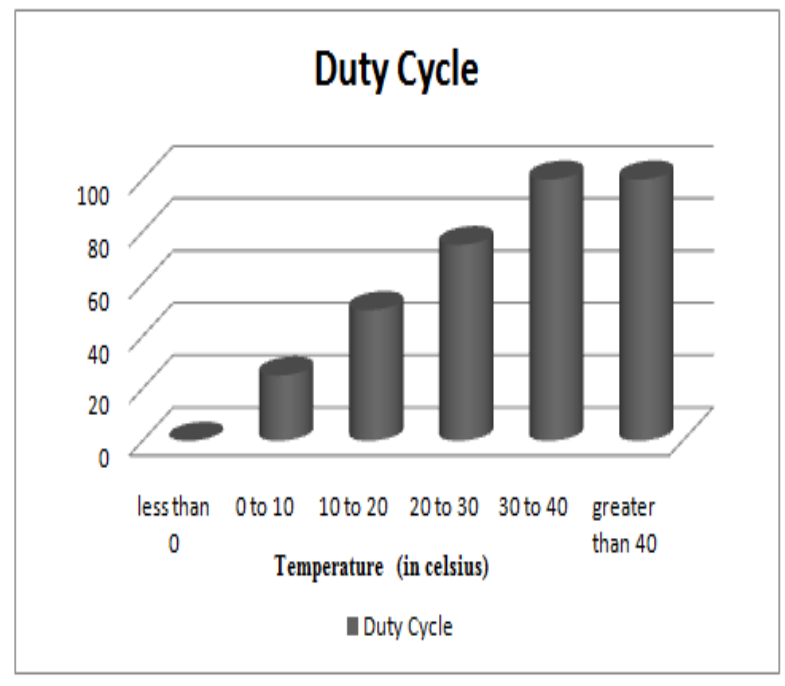

Fig.7. Temperature (in ${ }^{\circ} \mathrm{C}$ ) vs Duty Cycle

Variation of duty cycle with temperature (in Fahrenheit) has also been shown in Fig 8. The duty cycle is varied according to the room temperature and speed is controlled accordingly.

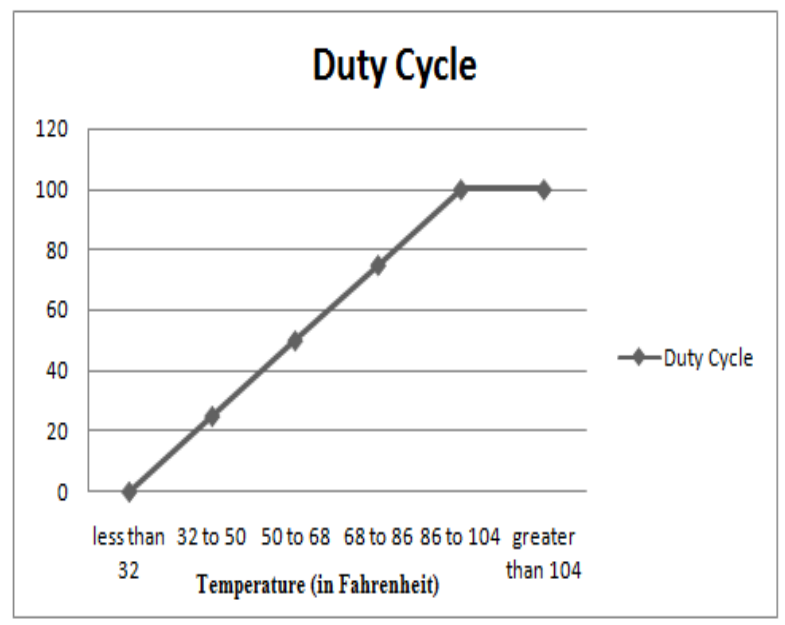

Fig.8. Temperature (in ${ }^{0}$ F) vs. Duty Cycle

\section{FLOWCHART OF THE SYSTEM}

The logical representation of the software code has been presented in the flowchart form. Fig 9 shows the flowchart of the logic implemented in the modeled system. The temperature is read from the temperature sensor and the condition is checked and the following processes are done:

1. When temperature is greater than zero and less than 10 degree Celsius, the fan speed is slow.

2. When temperature is greater than 10 and less than 20 degree Celsius, the fan speed is medium.

3. When temperature is greater than 20 and less than 30 degree Celsius, the fan speed is fast.

4. When temperature is greater than 30 and less than 40 degree Celsius, the fan speed is very fast.

5. When temperature is less than zero degree Celsius, the fan stops. 


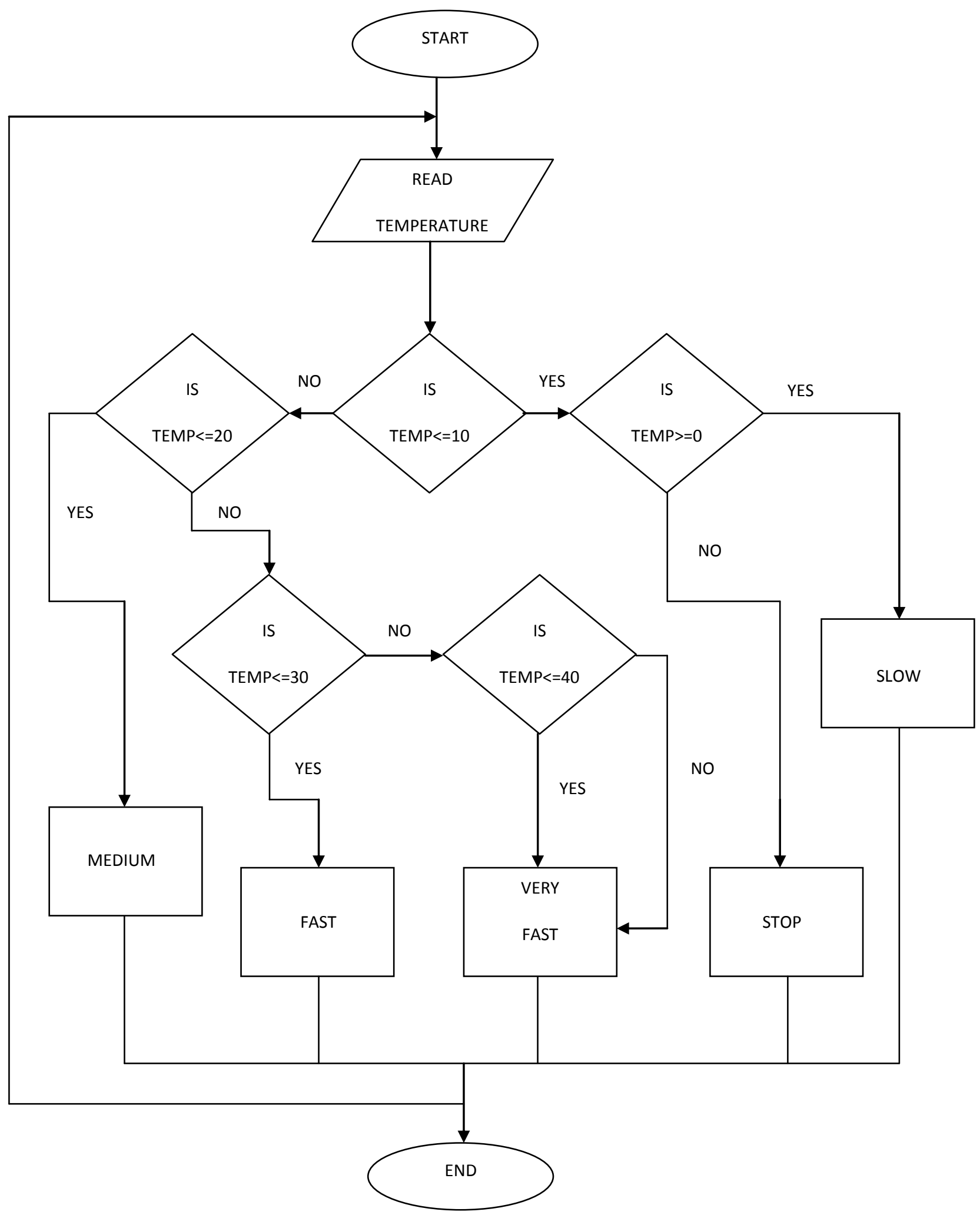

Fig.9. Flowchart of the System

\section{RESULTS}

The speed of the fan has been controlled using PWM technique according to the room temperature. The simulation of the system has been done on Proteus Professional v 8.0 software packages and it is running in good agreement .The logic used in the system is verified and shown in the flowchart form. The duty cycle is varied according to room temperature and speed of the fan is controlled accordingly. The graphs 
showing the relationship between duty cycle and the room temperature are plotted in MATLAB and accuracy of the system is validated. The design of the system presented in this paper is appropriate according to the modern technology.

\section{CONCLUSIONS AND FUTURE SCOPE}

A novel design of speed control of fan based on room temperature using PWM technique is proposed in this paper. The simulation of the system is working properly and the design is appropriate according to the modern needs and technology. The speed of fan depends on the room temperature and there is no need for regulating the speed manually. Various graphs have been plotted to show the varying relationships between different parameters. PWM technique is found to be appropriate for controlling fan speed according to room temperature. This design can be further extended in terms of area and power at layout and characteristic level by using Advanced VLSI applications.

\section{REFERENCES}

[1] Vaibhav Bhatia and Pawan Whig, "A Secured Dual Tone Multifrequency Based Smart Elevator Control System," International Journal of Research in Engineering and Advanced Technology, Volume 1, Issue 4, Aug-Sept, 2013.

[2] F. Luo, X. Zhao, and Y. Xu, "A new hybrid elevator group control system scheduling strategy based on particle swarm simulated annealing optimization algorithm", Intelligent Control and Automation (WCICA), 2010, pp. 5121-5124.

[3] T.-H Hsu, J.-F. Liu, P.-N Yu, W.-S. Lee, and J.-S. Hsu, "Development of an automatic parking system for vehicle," in Proc. of the International Conference on the
IEEE Vehicle Power and Propulsion, Sept. 3-5 Harbin, China, 2008, pp. 1-6.

[4] I. Panagopoulos, C. Pavlatos and G. Papakonstantinou, "An Embedded Microprocessor for Intelligent Control," Journal of Intelligent and Robotic Systems. Springer Netherlands, vol. 42, 2005, pp. 179-211.

[5] Md. M. Islam, F. H. Md. Rafi, A. F. Mitul and M. Ahmad, "Development of a Noninvasive Continuous Blood pressure Measurement and Monitoring system", Proceedings of the International conference on ICIEV, May- 2012,pp. 1085-1090.

[6] S. Kwakyea and A. Baeumner"An embedded system for portable electrochemical detection", Sens. Actuat. B, vol. 123, no. 1, 2007, pp.336 -343.

[7] L. J. Fu"Remote control system using infrared technology based on PIC16F873 microcontroller", Electron. Technol., vol. 45, no. 10, 2008, pp.68 - 70 .

[8] B. Ismail, S. Taib, A. R. M. Saad, M. Isa and C. M. Hadzer, "Development of a Single Phase SPWM Microcontroller-Based Inverter", Proceedings of the Annual International Conference of the PECon, November 2006,pp. 437-440.

[9] Xiaodong Xia, Based on Single Chip MicrocomputerRemote Wireless Control System Design. Coal Mine Machinery, vol. 32 (8), 2011, pp. 202-204.

[10] T. R. F Fulford-Jones, W. Gu-Yeon, and M Welsh, "A portable, low power, wireless two-lead EKG system," Proceedings of the 26th Annual International Conference of the IEEE Engineering in Medicine and Biology Society (EMBC-04), 1-5 Sept. 2004 , pp.2141-2144. 